\title{
Article \\ Explainable Artificial Intelligence-Based Decision Support System for Assessing the Nutrition-Related Geriatric Syndromes
}

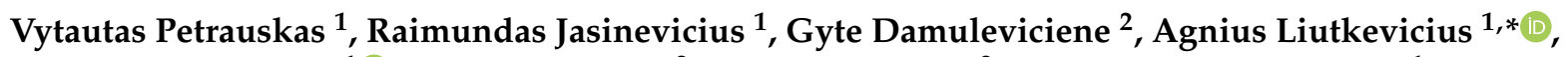 \\ Audrone Janaviciute ${ }^{1}$, , Vita Lesauskaite ${ }^{2}$, Jurgita Knasiene ${ }^{2}$, Zygimantas Meskauskas ${ }^{1}$, Juozas Dovydaitis ${ }^{1}$, \\ Vygintas Kazanavicius $^{1}$ and Raminta Bitinaite-Paskeviciene ${ }^{2}$ \\ 1 Centre of Real Time Computer Systems, Kaunas University of Technology, 44249 Kaunas, Lithuania; \\ vytautas.petrauskas@ktu.lt (V.P.); raimundas.jasinevicius@ktu.lt (R.J.); audrone.janaviciute@ktu.lt (A.J.); \\ zygimantas.meskauskas@ktu.lt (Z.M.); juozas.dovydaitis@ktu.lt (J.D.); vygintas.kazanavicius@ktu.lt (V.K.) \\ 2 Clinical Department of Geriatrics, Lithuanian University of Health Sciences, 44307 Kaunas, Lithuania; \\ gyte.damuleviciene@lsmuni.lt (G.D.); vita.lesauskaite@lsmuni.lt (V.L.); jurgita.knasiene@lsmuni.lt (J.K.); \\ raminta.bitinaite@lsmuni.lt (R.B.-P.) \\ * Correspondence: agnius.liutkevicius@ktu.lt; Tel.: +370-626-22-304
}

check for updates

Citation: Petrauskas, V.; Jasinevicius,

R.; Damuleviciene, G.; Liutkevicius,

A.; Janaviciute, A.; Lesauskaite, V.;

Knasiene, J.; Meskauskas, Z.;

Dovydaitis, J.; Kazanavicius, V.; et al.

Explainable Artificial

Intelligence-Based Decision Support

System for Assessing the

Nutrition-Related Geriatric

Syndromes. Appl. Sci. 2021, 11, 11763.

https://doi.org/10.3390/

app112411763

Academic Editor: Keun Ho Ryu

Received: 19 October 2021

Accepted: 8 December 2021

Published: 10 December 2021

Publisher's Note: MDPI stays neutral with regard to jurisdictional claims in published maps and institutional affiliations.

Copyright: (c) 2021 by the authors. Licensee MDPI, Basel, Switzerland. This article is an open access article distributed under the terms and conditions of the Creative Commons Attribution (CC BY) license (https:// creativecommons.org/licenses/by/ $4.0 /)$.

\begin{abstract}
The use of artificial intelligence in geriatrics is very promising and relevant, as the diagnosis of a geriatric patient is a complex, experience-based, and time-consuming process that involves a variety of questionnaires and subjective and inaccurate patient responses. This paper proposes the explainable artificial intelligence-based (XAI) clinical decision support system (CDSS) to assess nutrition-related factors (symptoms) and to determine the likelihood of geriatric patient health risks associated with four syndromes: malnutrition, oropharyngeal dysphagia, dehydration, and eating disorders in dementia. The proposed system's prototype was tested under real conditions at the geriatric department of Lithuanian University of Health Sciences Kaunas Hospital. The subjects of this study were 83 geriatric patients with various health conditions. The assessments of the nutritional status and syndromes of the patients provided by the CDSS were compared with the diagnoses of the physicians obtained using standard assessment methods. The results show that proposed CDSS can efficiently diagnose nutrition-related geriatric syndromes with high accuracy: $87.95 \%$ for malnutrition, $87.95 \%$ for oropharyngeal dysphagia, $90.36 \%$ for eating disorders in dementia, and $86.75 \%$ for dehydration. The research confirms that the proposed XAI-based CDSS is an effective tool, able to assess nutrition-related health risk factors and their dependencies and, in some cases, makes even a more accurate decision than a less experienced physician.
\end{abstract}

Keywords: explainable artificial intelligence (XAI); computing with words (CWW); clinical decision support system (CDSS); geriatric syndrome; fuzzy logic-based reasoning

\section{Introduction}

Geriatricians are constantly faced with the problem of assessing the health status of patients while diagnosing diseases and syndromes because they must take into account multiple interrelated symptoms (health risk factors). As in any field of medicine, the quality of a patient's diagnosis depends directly on the physician's experience and ability to make a thorough assessment of the patient's health status. In geriatrics, this problem is relevant because current generally accepted methods for assessing the health status of patients are based on a wide variety of assessment tools, including daily activity evaluation, depression screening, dementia screening, initial malnutrition screening, assessment of the risk of bedsores, risk of falls, somnolence, swallowing disorder, etc. Therefore, the less experienced geriatrician is faced with the problem of which assessment methods should be used in the concrete case, as well as to decide the level of detail of the assessment. Symptoms and syndromes associated with nutritional disorders pose the greatest health risk to the elderly and affect other disorders and threats (e.g., frailty, falls, etc.) as described 
in [1,2]. Geriatric syndromes, associated with poor nutritional status, were chosen for this study because malnutrition in older adults is associated with poor health outcomes, adverse effects on physical, psychological, and social aspects of health [3]. The effects of poor nutrition and diet can lead to a variety of senior health concerns [4], including:

- A higher risk of hospitalization;

- An increased risk of death;

- A weakened immune system, which increases the risk of infections and cancer;

- Poor wound healing;

- Muscle weakness and decreased bone mass, which can lead to falls and fractures;

- Depression;

- Decreased cognitive function;

- Functional decline;

- Social isolation;

- Decreased quality of life.

Not only can the nutritional state influence health disorders, but also, once the disease starts, it can affect the nutritional status as well; thus, the connection is reciprocal. On the other hand, health professionals do not pay enough attention to nutritional status and even do not have sufficient knowledge of how to diagnose and control it [5]. During the nutritional status assessment, the physician interviews the patient in an attempt to determine what typical symptoms are relevant to that individual and then, using standardized assessment rules, attempts to predict which diseases or syndromes correspond to those symptoms. The diagnostic process is complicated by the fact that in certain cases, the same symptoms are used to diagnose different diseases or syndromes. Therefore, it is necessary to assess the degree of severity of the symptom, which is hardly measurable in common cases. Furthermore, the syndromes are interrelated and influence each other, and for an inexperienced physician (e.g., junior resident), it is difficult to assess which disease or their combination is more specific to the patient. This means that it is not sufficient to use computerized questionnaires for the detection of geriatric syndromes alone because these questionnaires use inaccurate and subjective estimates of symptoms (usually a 1-4 point scale). In order to make the diagnosis as accurate as possible, it is not enough to simply estimate the questionnaires, but a clinical decision support system (CDSS) is needed to assess the interdependencies between symptoms and syndromes, as well as some uncertainties arising from subjective survey data.

This paper presents the software implementation and evaluation of the clinical decision support system (CDSS) for the geriatric department of Lithuanian University of Health Sciences Kaunas Hospital, which solves the previously mentioned problems related to the geriatric diagnosis process, allowing even less experienced medical personnel to make more accurate decisions faster and easier. The prototype of CDSS is based on the original explainable artificial intelligence (XAI)-based decision support model presented in the previous scientific work [6] published by the authors of this paper. The proposed model was implemented in the CDSS prototype tested under real conditions at the geriatric department of Lithuanian University of Health Sciences Kaunas Hospital. It was used to assess nutrition-related factors (symptoms) and to evaluate the likelihood of four syndromes: malnutrition, oropharyngeal dysphagia, dehydration, and eating disorders in dementia.

Many medical institutions use computer-based systems to manage and process medical data. Some of these systems, namely clinical decision support systems (CDSS), are helpful tools for medical personnel to automate decision making and diagnose various health disorders with more precision. CDSS is widely used in certain medical fields, including the diagnoses of heart diseases [7-10], kidney diseases [11,12], diabetes [13,14], cancer [15,16], Alzheimer's disease [17], etc. CDSS are also applied in the field of geriatrics, where they are used successfully to assess signs of depression with an average accuracy of $95 \%$ [18] and diagnose dementia with a balanced accuracy of $82.3 \%$ [19], with an accuracy of $95.2 \%$ [20], and with an accuracy varying from $73 \%$ to $92 \%$ for various cases as presented in [21]. 
All the papers we analyze apply a variety of artificial intelligence techniques, such as neural networks, support vector machines, Bayes classifiers, or fuzzy logic and fuzzy cognitive maps. Fuzzy logic-based methods are quite popular for CDSS implementation, especially when uncertain input data should be processed as presented in [12,22]. The authors of [12] demonstrated that their proposed fuzzy DSS outperformed the physicians in diagnosing kidney stones and kidney infection and achieved 95\% and $85 \%$ accuracy, respectively, while physicians achieved only $70 \%$ and $65 \%$ accuracy in the same cases. The authors of [22] proposed an automatic computer-aided diagnosis system based on a fuzzy cognitive map (FCM) to determine the grade of celiac disease and achieved a classification accuracy of $91.01 \%$.

As stated by [23], fuzzy modeling has a huge potential for the development of advanced explainable AI (XAI) systems, in which the results of the solution can be understood by humans. Fuzzy-based XAI enables the development of decision-making rules based on the knowledge of medical experts and is capable of assessing inaccurate and subjective input data. XAI-based decision support systems are suitable for the diagnosis and evaluation of patient health risks when the decision is determined by many different interdependent factors. Furthermore, XAI-based CDSS allows verifying the reason behind the decision suggested by the system and comparing this result with the physician's reasoning. Therefore, the physician can be sure that the decision proposed by the system is the right one, especially in cases where the physician has a different initial opinion. The research presented in this work contributes to the field of the application of artificial intelligence in CDSS systems. This paper describes the application of explainable artificial intelligence (XAI) for the implementation of the CDSS to diagnose geriatric diseases and syndromes related to malnutrition. Studies related to the diagnosis of nutritional disorders and the identification of related syndromes using artificial intelligence and CDSS are not found in the scientific literature, except our initial study [6], which proposes a theoretical XAI-based decision support model for nutrition-related geriatric disorders.

This paper presents the real-world experimental evaluation of the theoretical XAIbased CDSS model [6] with real patient data. The aim of this study is to develop the software implementation of web-based CDSS and to evaluate the effectiveness of the proposed system by comparing its diagnoses with those of medical experts. This tool would be useful for the inexperienced physician (resident geriatricians, family physicians, general practitioners, etc.) to pay attention to nutrition-related geriatric syndromes and perform a detailed examination of nutrition-related disorders.

\section{Materials and Methods}

\subsection{Participants}

The patients were randomly selected for this study (every second patient hospitalized in the geriatric department of the Kaunas Hospital of the Lithuanian University of Health Sciences was enrolled). Patients were hospitalized primarily through urgent admission due to pyelonephritis, pneumonia, and syncope and through planned admission due to dysphagia, malnutrition, dizziness, Parkinson's disease, dementia, and falls. A total of 90 patients were invited to participate in this study, five of whom were excluded from the study according to exclusion criteria. Furthermore, two patients refused to continue participating due to physical exhaustion. In total, 83 geriatric patients participated in this study. The average age of the participants was 81.5 years, and $55(66.2 \%)$ of the patients were women. The study inclusion criteria were as follows: patients hospitalized in the geriatric department, ability to understand and speak Lithuanian, and signature of informed consent. The study exclusion criteria were as follows: deafness, advanced dysphagia requiring enteral nutrition, advanced dementia or cancer, and terminal renal, cardiac, pulmonary, or hepatic insufficiency. 


\subsection{Experimental Procedure}

Prior to any test or intervention and before being evaluated for the study's inclusion criteria, all participants signed a written informed consent approved by the Regional Bioethics Committee at the Lithuanian University of Health Sciences (no. BE-2-106).

As shown in Figure 1, the evaluation of the proposed CDSS was performed by researchers, resident geriatricians, and expert geriatricians. The researcher's role was taken by doctoral students, who interviewed the patients and entered data into the CDSS. In parallel, resident geriatricians performed diagnostic procedures using comprehensive geriatric assessment methods and laboratory examinations. These procedures were repeated every day within a 10-day period until the final diagnosis was made. The professors of the Clinical Department of Geriatrics of the Lithuanian University of Health Sciences played the role of experts, who reviewed the diagnoses of resident geriatricians, performed additional assessments and laboratory examinations to make the final diagnoses. The efficiency of the proposed CDSS was evaluated by comparing its diagnoses with the diagnoses of resident geriatricians and expert geriatricians (considered the gold standard). The decision-making rules of the proposed CDSS are implemented using fuzzy logic-based XAI, which adapts a set of questionnaires composed according to international practice and supplemented by knowledge accumulated in the Clinical Department of Geriatrics of the Lithuanian University of Health Sciences. In order to assess malnutrition, oropharyngeal dysphagia, eating disorders in dementia, and dehydration, the MNA-SF scale [24], the dysphagia screening tool EAT-10 [25], Edinburgh Feeding Evaluation in Dementia Scale [26], and a set of 10 questions based on the ESPEN guideline on clinical nutrition and hydration in geriatrics [1] were used.

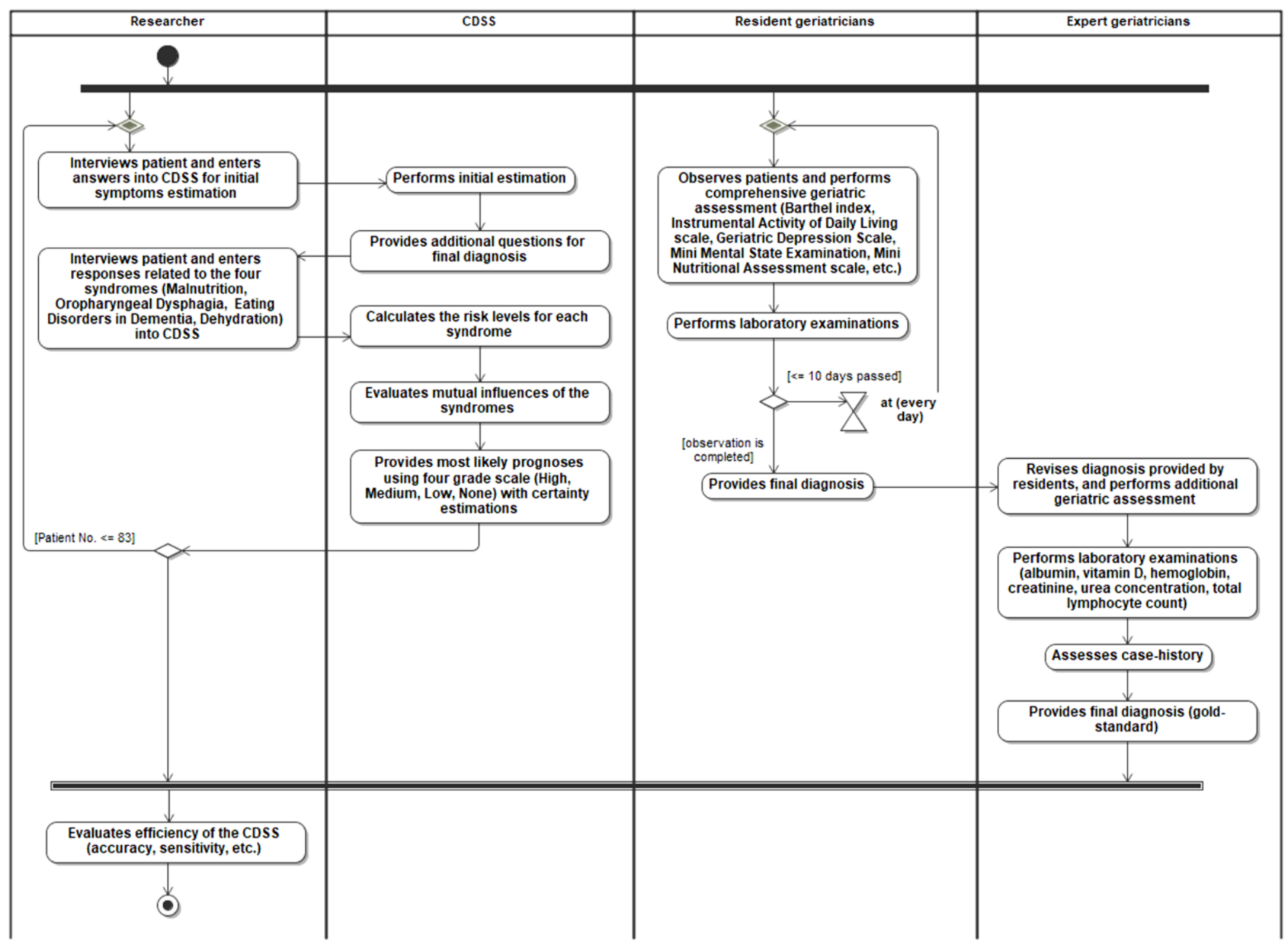

Figure 1. The procedure of experimental evaluation of the proposed CDSS. 


\subsection{Enhanced SWOT Maps and Fuzzy Expert Maps for Risk Evaluation}

This paper presents a new universalized concept of a system using a dynamic SWOT analysis network called a fuzzy SWOT map (FSM) for fuzzy risk control in complex environments and a fuzzy cognitive map (FCM)-type network for the aggregation of appropriate leverages, recommendations, or even immediate actions to be taken that would enable improvement of the situation. That part of the system is called LEVERAGE for simplicity and is also built on the fuzzy cognitive maps (FCM)-type network consisting of elements operating on the expert knowledge presented in the form of the lists of IF ... THEN-type fuzzy logic rules. Such a network is called fuzzy expert maps (FEM) [27], and its philosophy fully covers the functioning of many real systems, including clinical decision support systems. A generalized view of such an abstract system is shown in Figure 2.

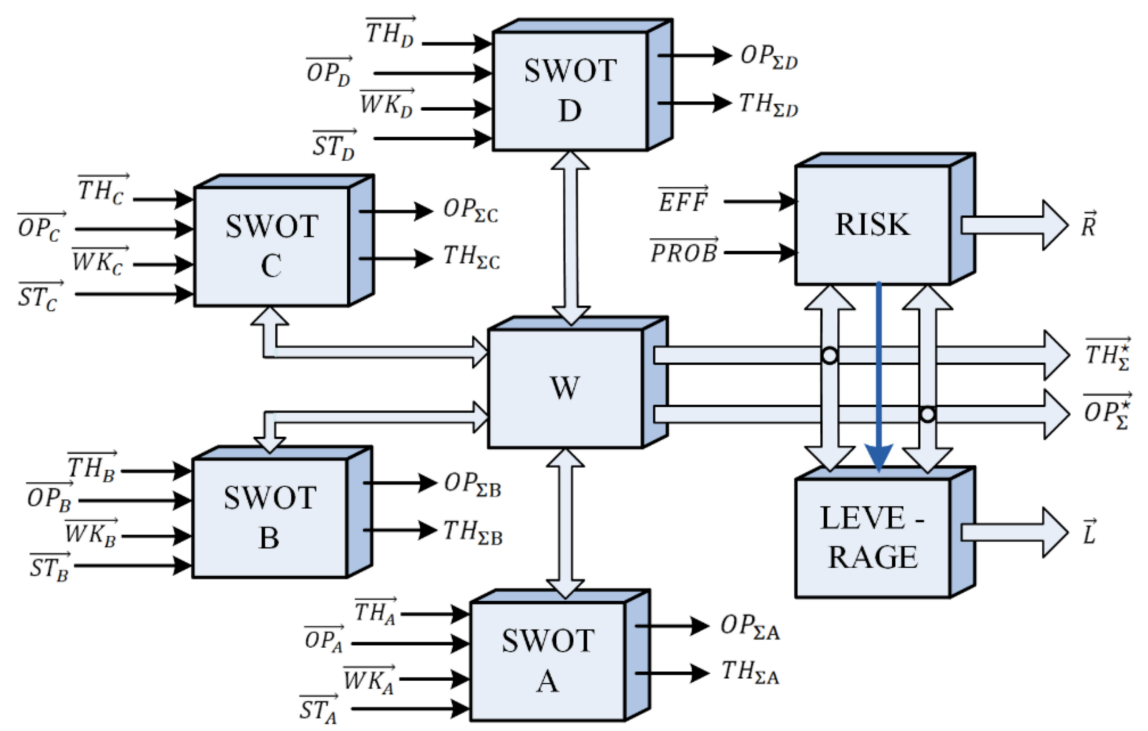

Figure 2. A generalized view of an abstract risk analysis and decision support system based on XAI.

Figure 2 shows an example of a conceptual architecture of the XAI-based risk analysis and decision support system for the evaluation of four situations (for example, specific diseases or syndromes). Depending on the application, the number of situations can vary. The architecture presented in Figure 2 includes SWOT analysis subsystems for each of four situations (A, B, C, and D), interacting using the FSM network methodology. Here, $\mathrm{W}$ is a matrix that describes interactions of situations, the coefficients of which verbally or numerically (after defuzzification of words) express the nature and strengths of those interactions in the interval $[-1,+1]$. Each situation $e \in\{A, B, C, D\}$ usually is characterized by four SWOT vectors $\overrightarrow{S T_{e}}, \overrightarrow{W K_{e}}, \overrightarrow{O P_{e}}, \overrightarrow{T H_{e}}$ also representing verbally or numerically (after the defuzzification of words) the strength and weaknesses of the situation under investigation as well as its opportunities and threats correspondingly. The summarized and normalized threats $\overrightarrow{T H_{\Sigma}^{\star}}$ and summarized and normalized opportunities $\overrightarrow{O P_{\Sigma}^{\star}}$ are used to evaluate the possible level of risk $\overrightarrow{\mathrm{R}}=\left(R_{A}, R_{B}, R_{C}\right.$, and $\left.R_{D}\right)$ and to suggest and recommend a certain set of possible activities $\overrightarrow{\mathrm{L}}=\left(L_{1}, L_{2}, \ldots, L_{l}, \ldots, L_{L}\right)$ (leverages from the arsenal of available activities) to be performed, enabling the improvement of performance in the situation or project under consideration.

Generally, a measurable level of risk can be calculated as a value of a certain function $\mathrm{R}$, depending on the level of threats $\overrightarrow{T H_{\Sigma}^{\star}}$, opportunities $\overrightarrow{O P_{\Sigma}^{\star}}$ efforts dedicated to systems development $\overrightarrow{E F F}$ and the probabilities of corresponding events $\overrightarrow{P R O B}$, as proposed and shown in the work of [28]: $R=R(E F F \uparrow ; O P \downarrow ; T H \uparrow ; P R O B \downarrow)$. The arrows $\uparrow$ and $\downarrow$ mean 
increase and decrease in $\mathrm{R}$, respectively. The general idea of the structure of the functional organization for the risk evaluation engine is presented in Figure 3.

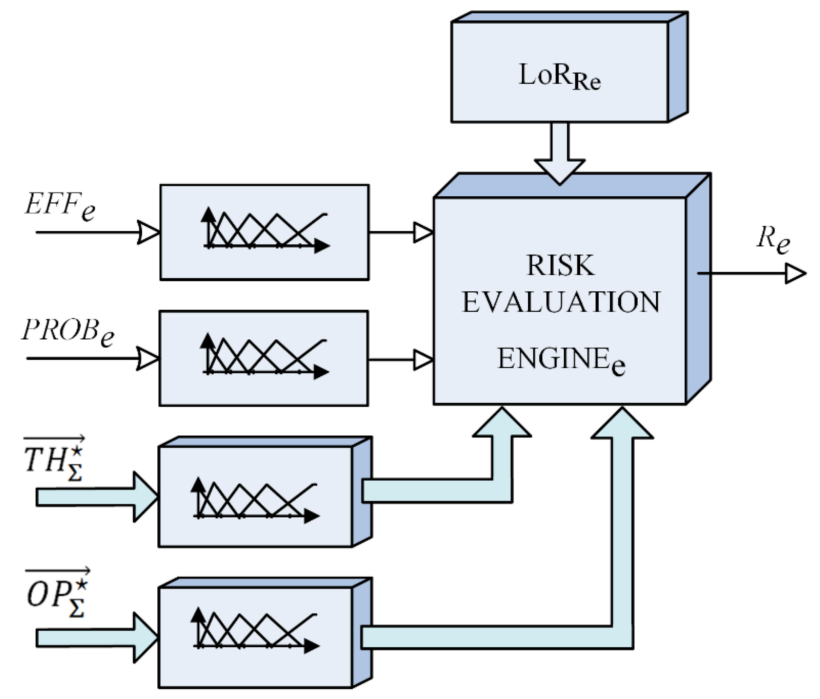

Figure 3. Risk evaluation element of the fuzzy expert map (FEM).

The earlier presented informal reasoning is prescribed by an IF ... THEN-type list or fuzzy rules $\left(\mathrm{LoR}_{\mathrm{Re}}\right)$ is drafted by experts based on their knowledge. Such a structure is called FEM, the fuzzy expert map as it was proposed in [27] and mentioned above.

\subsection{XAI-Based CDSS as a Partial Case of the Universalized FSM}

The functional organization of a structure for the XAI-based clinical decision support system, proposed in [6], is the partial cause of the concept of the universalized fuzzy SWOT maps (FSM) network presented and developed in Section 2.3. The model of the CDSS presented in [6] takes into account four mutually influencing syndromes such as MALNUTRITION (A), OROPHARYNGEAL DYSPHAGIA (B), DEHYDRATION (C), and EATING DISORDERS IN DEMENTIA (D). They are considered as situations: A, B, C, and D. Their mutual influence is presented in Figure 4 and verbally and/or numerically is represented by matrix $\mathrm{W}$ as it is shown in Figure 2 (thickness of the arrows represents the strength of influence).

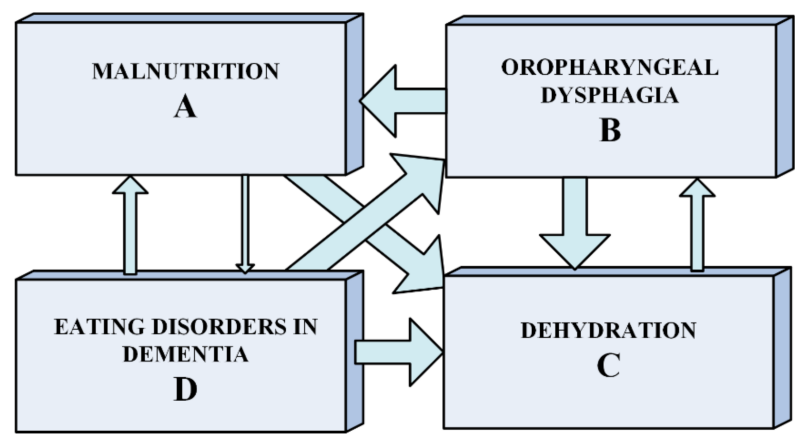

Figure 4. Mutually influencing syndromes.

It should be stressed that the model described in [6] uses only threats $\overrightarrow{T H}_{e}$ and weaknesses $\overrightarrow{W K_{e}}$ as the inputs to corresponding SWOT elements, where $e \in\{A, B, C, D\}$ (see Figure 2). In this simplified case, each SWOT element summarizes and normalizes the assessments of the symptoms of a particular syndrome as threats $\left(\overrightarrow{T H_{e}}\right)$ in numerical and/or verbal form, and summarizes and normalizes the responses to the questionnaire 
for each syndrome as weaknesses $\left(\overrightarrow{W K_{e}}\right)$ specific to that syndrome and is also processed numerically and/or verbally. After performing a SWOT analysis and taking into account the mutual influence of the syndromes, the FSM network produces a summarized output result as a collection of threats levels of each syndrome (A, B, C, and D) composed in the vector $\overrightarrow{T H_{\Sigma}^{\star}}$. This vector is suitable for a proper verbalization, usually called a diagnosis, and presenting not only numerical evaluation of the situation under consideration but also understandable to human verbal description, followed by some reasoning about the certainty value of the decision.

An example of the diagnosis produced by a proposed XAI-based CDSS model is given in Table 1. Here we have the pessimistic, optimistic, and the most likely diagnosis for one patient and the values of certainties of the proposed decisions.

Table 1. An example of diagnostic advice produced by the model of XAI-based CDSS for one patient.

\begin{tabular}{ccccc}
\hline & \multicolumn{4}{c}{ General Threat of Syndromes (Threat, Certainty) } \\
\cline { 2 - 5 } & $\begin{array}{c}\text { Malnutrition } \\
(\mathbf{M})\end{array}$ & $\begin{array}{c}\text { Oropharyngeal } \\
\text { Dysphagia (OD) }\end{array}$ & $\begin{array}{c}\text { Eating Disorders } \\
\text { in Dementia (D) }\end{array}$ & $\begin{array}{c}\text { Dehydration } \\
\text { (DH) }\end{array}$ \\
\hline Pessimistic & LARGE & MEDIUM & SMALL & LARGE \\
diagnosis & 0.228 & 0.396 & 0.560 & 0.475 \\
\hline Optimistic & MEDIUM & SMALL & NOT & MEDIUM \\
diagnosis & 0.772 & 0.604 & 0.440 & 0.525 \\
\hline Most likely & MEDIUM & SMALL & SMALL & MEDIUM \\
diagnosis & 0.772 & 0.604 & 0.560 & 0.525 \\
\hline
\end{tabular}

\subsection{CDSS Implementation}

The decision support system for assessing the nutrition-related geriatric syndromes was implemented using the previously discussed XAI-based risk assessment model and system concept. This CDSS was deployed at the clinical department of geriatrics of Lithuanian University of Health Sciences (accessible online: https:/ / gerimodis.lt, accessed on 9 December 2021), where it is used by physicians with different levels of experience in geriatrics.

The proposed system uses screening questions for four geriatric syndromes (Figure 5) and medically approved questionnaires (Figure 6). Numerical and/or verbal symptoms estimates and total estimates of the answers to the questions in each questionnaire serve as input data for the CDSS. The diagnosis process begins with answering screening questions regarding four nutrition-related geriatric syndromes. Each syndrome is defined by four characteristic questions (Figure 5). The CDSS takes into account the mutual influence of the syndromes and gives the initial prognostic answer, and then the specialist continues by filling out questionnaires related to syndromes (Figure 6). The questionnaires were selected according to international geriatric practice, adapted and complemented based on the expertise of the specialists of the clinical department of geriatrics of Lithuanian University of Health Sciences. The MNA-SF scale [25] was used for malnutrition (Figure 6a), the EAT-10 dysphagia screening tool [29] was used for oropharyngeal dysphagia (Figure 6b), the Edinburgh Feeding Evaluation in Dementia Scale [2] was used for dementia (Figure 6c), the set of questions formed using the ESPEN guidelines on clinical nutrition and hydration in geriatrics [1] was used for dehydration (Figure 6d). These questionnaires are given in Appendix A.

Since the CDSS is based on fuzzy logic, the scores provided by the questionnaires are converted into verbal estimates: "good", "might be bad", "bad" ("Model estimate" tables in Figure 6). For the MNA-SF scale, good nutritional status corresponds to 0-2 points, suspicion of malnutrition corresponds to 3-6 points, and malnutrition corresponds to 7-14 points. Please note that the MNA-SF scale used in the CDSS is modified compared to the original one, presented in the work of [25]. The modified scale has an inverted 
scoring system, where malnutrition is indicated by a higher score. For the EAT-10 dysphagia screening tool, the absence of the disorder corresponds to $0-2$ points, the suspected dysphagia is indicated by 3-10 points, and the dysphagia corresponds to $11-40$ points. For the Edinburgh feeding evaluation in the dementia scale, the absence of eating disorder in dementia corresponds to $0-5$ points, the suspected eating disorder in dementia is indicated by $6-10$ points, and eating disorder in dementia corresponds to $11-20$ points. For dehydration, an original set of questions was formed using the ESPEN guidelines on clinical nutrition and hydration in geriatrics. The absence of dehydration corresponds to $0-5$ points, the possible dehydration is indicated by $6-10$ points, and the dehydration corresponds to $11-40$ points.

\begin{tabular}{|c|c|c|}
\hline & Symptom & Estimation (0-3) \\
\hline \multirow{4}{*}{ M } & Decreased appetite & 2 \\
\hline & Eats less & 2 \\
\hline & Weight loss & 2 \\
\hline & Clothes, shoes, rings became too big & 0 \\
\hline \multirow{4}{*}{ OD } & Chokes while eating or drinking & 0 \\
\hline & Coughing while eating or drinking & 0 \\
\hline & Voice changes after drinking & 0 \\
\hline & It's difficult to swallow the pills & 0 \\
\hline \multirow{4}{*}{ D } & Refuses to open mouth & 0 \\
\hline & Food spills and falls out of the mouth & 0 \\
\hline & Spits out food & 0 \\
\hline & No longer able to eat & 0 \\
\hline \multirow{4}{*}{ DH } & Drinks small amount of fluids & 3 \\
\hline & Urine became darker & 3 \\
\hline & Less urination & 3 \\
\hline & Dry mouth & 3 \\
\hline
\end{tabular}

Figure 5. Screenshot of the CDSS initial symptoms estimation questionnaire. Here: M-malnutrition, OD—oropharyngeal dysphagia, D—eating disorders in dementia, $\mathrm{DH}$-dehydration.

\begin{tabular}{|c|c|c|c|c|}
\hline & & & & Estimation \\
\hline \multicolumn{4}{|c|}{$\begin{array}{l}\text { 1. Has there been a decrease in food intake due to loss of appetite due to indigestion, } \\
\text { chewing, or swallowing in the last } 3 \text { months? }\end{array}$} & 1 \\
\hline \multicolumn{4}{|c|}{ 2. Weight loss in the last 3 months } & 2 \\
\hline \multicolumn{4}{|l|}{ 3. Physical activity } & 2 \\
\hline \multicolumn{4}{|c|}{$\begin{array}{l}\text { 4. The patient had experienced psychological stress or had an acute illness in the past } 3 \\
\text { months }\end{array}$} & 2 \\
\hline \multicolumn{4}{|c|}{ 5. Neuropsychological disorders } & 2 \\
\hline \multirow{2}{*}{$\mathrm{BMI}$ or $\mathrm{CC}$} & \multicolumn{3}{|l|}{ 6. BMI } & 1 \\
\hline & \multicolumn{3}{|c|}{ 7. Calf circumference (CC) } & 0 \\
\hline \multicolumn{4}{|l|}{ Total score } & 10 \\
\hline \multicolumn{4}{|c|}{ Maximum number of points } & $\begin{array}{l}14 \\
\text { (fourteen) }\end{array}$ \\
\hline & \multicolumn{3}{|c|}{ Model estimate } & \\
\hline & $0-2$ points & normal diet (good) & 0 & \\
\hline & 3-6 points & risk of $\mathrm{M}$ (might be bad) & 0.5 & \\
\hline & 7-14 points & M (bad) & 1 & \\
\hline
\end{tabular}

(a)

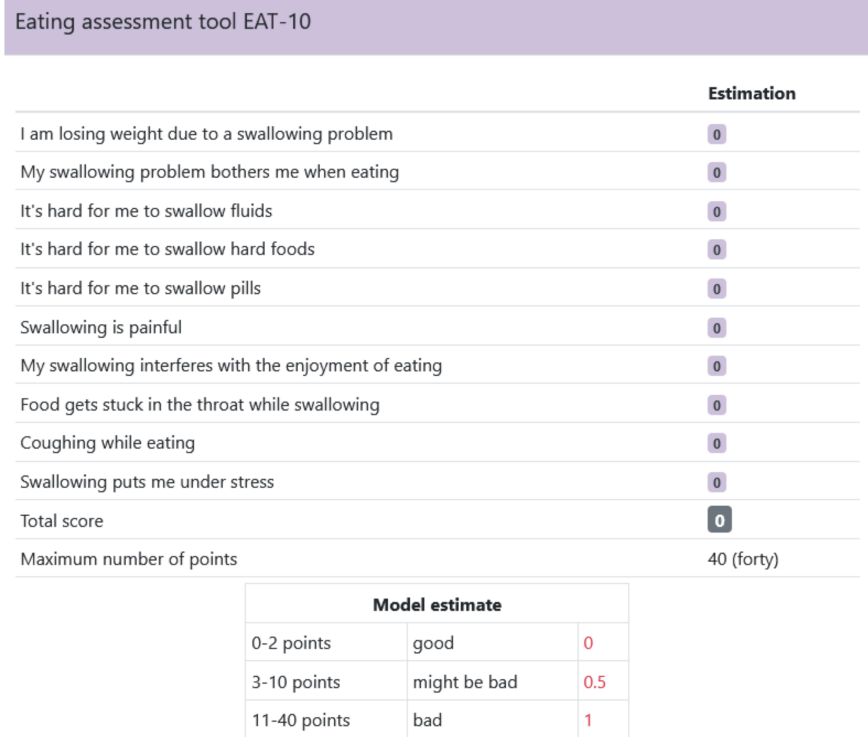

(b)

Figure 6. Cont. 


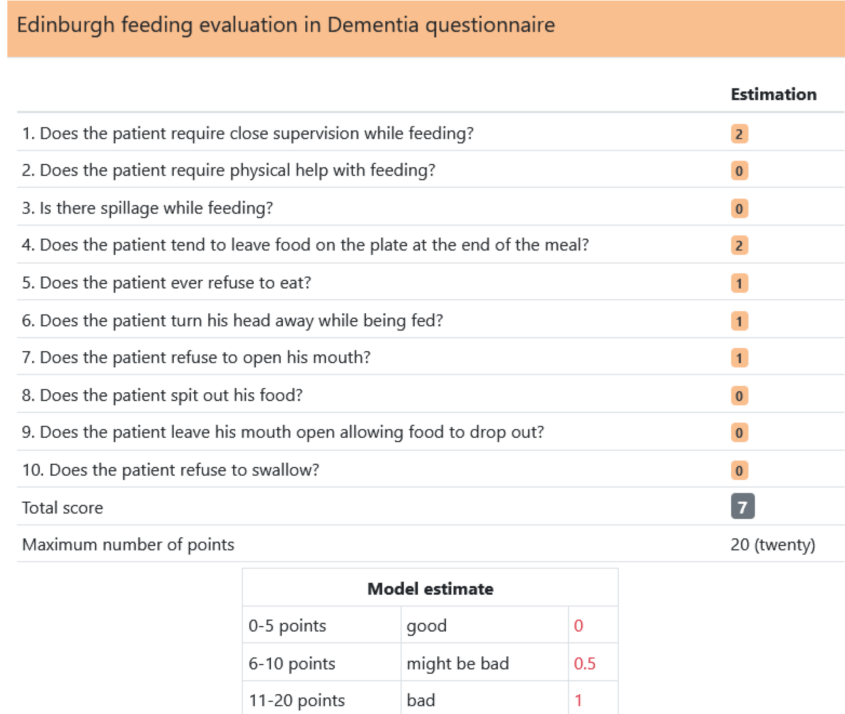

(c)
Dehydration assessment form

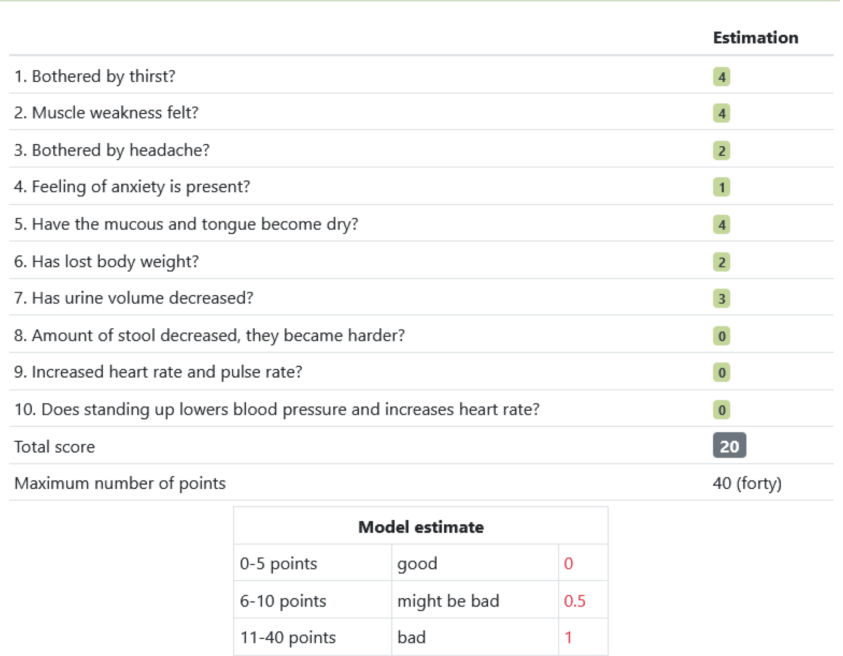

(d)

Figure 6. Screenshots of CDSS symptoms estimation questionnaires: (a) MNASF scale for malnutrition; (b) EAT-10 screening tool for oropharyngeal dysphagia; (c) Edinburgh Feeding Evaluation in Dementia Scale; (d) questionnaire formed using the ESPEN guidelines on clinical nutrition and hydration in geriatric dehydration.

The CDSS provides the specialist with a pessimistic, optimistic, or most likely prognosis of geriatric syndromes, as presented in Figure 7. The system gives the final prognosis for each syndrome based on the highest certainty.

Results:

Most likely prognoses:

"Eating disorders in dementia" - "Low" with certainty of 0.915

"Malnutrition" - "Medium" with certainty of 0.865

"Oropharyngeal Dysphagia" - "Low" with certainty of 0.747

"Dehydration" - "High" with certainty of 0.59

Conclusion and recommendations: -

\begin{tabular}{|c|c|c|c|c|}
\hline \multicolumn{5}{|c|}{ Risk Of Geriatric Nutritional Disorders } \\
\hline & $\begin{array}{c}\text { Malnutrition } \\
\text { M }\end{array}$ & $\begin{array}{l}\text { Oropharyngeal dysphagia } \\
\text { OD }\end{array}$ & $\begin{array}{c}\text { Eating disorders } \\
\text { in dementia } \\
\text { D }\end{array}$ & $\begin{array}{l}\text { Dehydration } \\
\text { DH }\end{array}$ \\
\hline $\begin{array}{l}\text { Pessimistic } \\
\text { prognosis }\end{array}$ & $\begin{array}{l}\text { MEDIUM with certainty of } \\
0.865\end{array}$ & $\begin{array}{l}\text { MEDIUM with certainty of } \\
0.253\end{array}$ & $\begin{array}{l}\text { MEDIUM with certainty of } \\
0.085\end{array}$ & $\begin{array}{l}\text { HIGH with certainty of } \\
0.59\end{array}$ \\
\hline $\begin{array}{l}\text { Optimistic } \\
\text { prognosis }\end{array}$ & $\begin{array}{l}\text { LOW with certainty of } \\
0.135\end{array}$ & $\begin{array}{l}\text { LOW with certainty of } \\
0.747\end{array}$ & $\begin{array}{l}\text { LOW with certainty of } \\
0.915\end{array}$ & $\begin{array}{l}\text { MEDIUM with certainty of } \\
0.41\end{array}$ \\
\hline $\begin{array}{l}\text { Most likely } \\
\text { prognosis }\end{array}$ & $\begin{array}{c}\text { MEDIUM with certainty of } \\
0.865\end{array}$ & $\begin{array}{c}\text { LOW with certainty of } \\
0.747\end{array}$ & $\begin{array}{c}\text { LOW with certainty of } \\
0.915\end{array}$ & $\begin{array}{c}\text { HIGH with certainty of } \\
0.59\end{array}$ \\
\hline
\end{tabular}

Figure 7. The CDSS screenshot of diagnostic advice.

In the example given in Figure 7, the CDSS suggests that "Eating disorders in dementia" and "Oropharyngeal dysphagia" syndromes have a low manifestation with very high certainty, while "Malnutrition" syndrome has a medium level of occurrence and "Dehydration" syndrome is evaluated as "High" threat level.

\section{Results}

The results of the diagnostic study consist of the health state assessments of 83 patients. Part of these results is presented as an example in Table 2. The table contains verbal diagnostic reports (high, medium, small, none) with their estimates of possible certainty in 
the range [0-1] provided by the CDSS. The assessments of the resident geriatricians and the expert geriatricians are presented in the form of 1 or 0 , which means that the syndrome is diagnosed or not. These data were used for further processing and estimation of the effectiveness of the CDSS using ROC analysis.

Table 2. Results of the assessments of the proposed CDSS, the resident geriatricians, and expert geriatricians; Mmalnutrition, OD—oropharyngeal dysphagia, D—eating disorders in dementia, DH—dehydration.

\begin{tabular}{|c|c|c|c|c|c|c|c|c|c|c|c|c|}
\hline \multirow{2}{*}{$\begin{array}{l}\text { Subject's } \\
\text { No. }\end{array}$} & \multicolumn{4}{|c|}{ Assessments of the Syndromes Given by the CDSS (Threat, Certainty) } & \multicolumn{4}{|c|}{$\begin{array}{l}\text { Assessments of the Syndromes } \\
\text { Given by Resident Geriatricians }\end{array}$} & \multicolumn{4}{|c|}{$\begin{array}{l}\text { Assessments of the Syndromes } \\
\text { Given by Expert Geriatricians }\end{array}$} \\
\hline & $\mathbf{M}$ & OD & $\mathbf{D}$ & DH & M & OD & $\mathbf{D}$ & DH & M & OD & D & DH \\
\hline 1 & Small (0.583) & Small (0.901) & None (0.86) & Medium (0.738) & 1 & 0 & 0 & 1 & 0 & 0 & 0 & 1 \\
\hline 2 & High (0.724) & Small (0.921) & Small (0.559) & Medium (0.971) & 1 & 0 & 0 & 1 & 1 & 0 & 0 & 1 \\
\hline 3 & Small (0.8) & Small (0.95) & None (0.901) & Medium (0.773) & 1 & 0 & 0 & 1 & 0 & 0 & 0 & 1 \\
\hline 4 & High (0.724) & Small (0.955) & Small (0.559) & Small (0.535) & 0 & 0 & 0 & 1 & 1 & 0 & 0 & 0 \\
\hline 5 & Medium (0.616) & Medium (0.835) & Small (0.719) & Medium (0.613) & 0 & 1 & 1 & 1 & 1 & 1 & 0 & 1 \\
\hline 6 & Medium (0.99) & Small (0.75) & None (0.749) & Medium (0.627) & 1 & 0 & 0 & 1 & 1 & 0 & 0 & 1 \\
\hline$\ldots$ & $\ldots$ & $\ldots$ & $\ldots$ & $\cdots$ & $\ldots$ & $\ldots$ & $\cdots$ & $\ldots$ & $\ldots$ & $\cdots$ & $\cdots$ & $\ldots$ \\
\hline 82 & Medium (0.677) & Medium (0.958) & Small (0.58) & Medium (0.794) & 0 & 1 & 0 & 0 & 1 & 1 & 0 & 1 \\
\hline 83 & Medium (0.605) & Small (0.669) & None (0.887) & Medium (0.605) & 0 & 0 & 0 & 1 & 1 & 0 & 0 & 1 \\
\hline
\end{tabular}

The study was conducted by geriatric physicians with different experiences: firstand second-year resident geriatricians and expert geriatricians (professors with more than 20 years of experience). Because the CDSS is intended to provide advice to an inexperienced geriatrician or general practitioner, the study assessed the precision of the diagnoses provided by the CDSS and resident physicians. The diagnoses of expert geriatricians are considered the gold standard in this case.

For evaluation of the performance of the proposed CDSS, receiver operating characteristics (ROC) analysis was used by calculating accuracy, sensitivity, and specificity. The threshold values for the ROC curves were selected according to the risk levels and their certainties provided by the CDSS. The lowest threshold value corresponds to "small risk with certainty 0.5 ", while the highest threshold value corresponds to "medium risk with certainty 1.0 ". For example, "medium risk with certainty 0.7 " threshold value means that medium risks with certainty more than 0.7 and high risks with any certainty indicate disorder (positive condition), while medium risks with certainty less or equal than 0.7, small risks with any certainty and no risks with any certainty are classified as negative condition (no disorder). as shown in Figure 8.

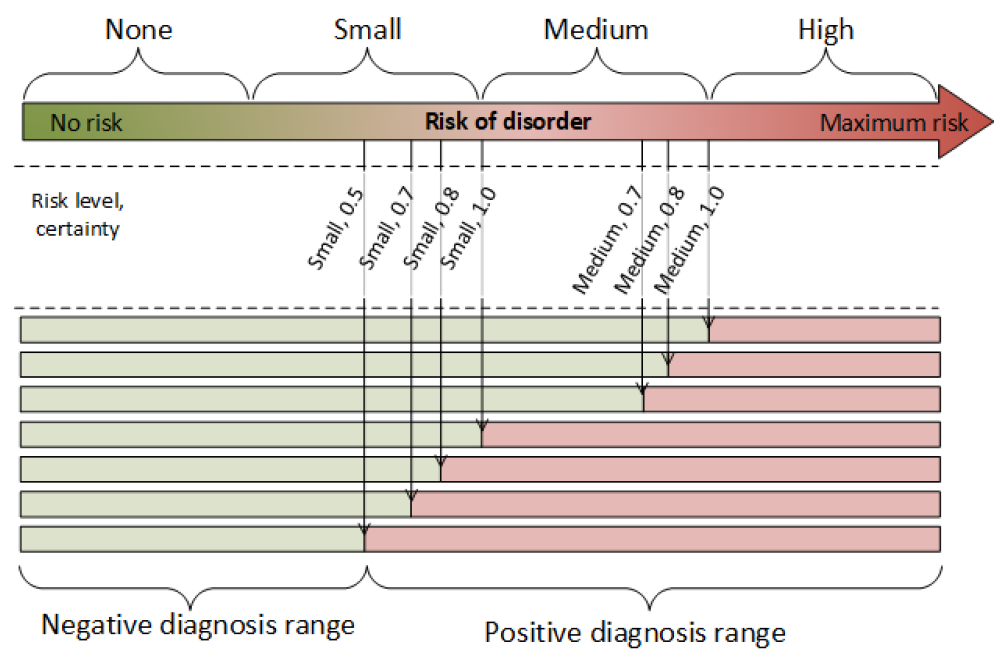

Figure 8. The threshold values for ROC analysis. Threshold values are defined by the risk level and its certainty. 
The results of the ROC analysis are presented in Table 3, which contains the percentage values of accuracy, sensitivity, and specificity, while Figure 9 represents the respective ROC curves for each syndrome (malnutrition, oropharyngeal dysphagia, eating disorders in dementia, dehydration).

Table 3. Performance evaluation of the CDSS and resident geriatricians.

\begin{tabular}{|c|c|c|c|c|c|c|c|c|c|c|c|c|c|}
\hline \multirow[b]{2}{*}{$\begin{array}{l}\text { Decision } \\
\text { Maker }\end{array}$} & \multirow[b]{2}{*}{$\begin{array}{l}\text { Threshold } \\
\text { Value (Risk, } \\
\text { Certainty) }\end{array}$} & \multicolumn{3}{|c|}{ Malnutrition } & \multicolumn{3}{|c|}{$\begin{array}{c}\text { Oropharyngeal } \\
\text { Dysphagia }\end{array}$} & \multicolumn{3}{|c|}{$\begin{array}{l}\text { Eating Disorders } \\
\text { in Dementia }\end{array}$} & \multicolumn{3}{|c|}{ Dehydration } \\
\hline & & $\begin{array}{c}\text { ACC } \\
(\%)\end{array}$ & $\begin{array}{l}\text { TPR } \\
(\%)\end{array}$ & $\begin{array}{c}\text { TNR } \\
(\%)\end{array}$ & $\begin{array}{c}\mathrm{ACC} \\
(\%)\end{array}$ & $\begin{array}{l}\text { TPR } \\
(\%)\end{array}$ & $\begin{array}{c}\text { TNR } \\
(\%)\end{array}$ & $\begin{array}{c}\text { ACC } \\
(\%)\end{array}$ & $\begin{array}{l}\text { TPR } \\
(\%)\end{array}$ & $\begin{array}{c}\text { TNR } \\
(\%)\end{array}$ & $\begin{array}{c}\mathrm{ACC} \\
(\%)\end{array}$ & $\begin{array}{l}\text { TPR } \\
(\%)\end{array}$ & $\begin{array}{c}\text { TNR } \\
(\%)\end{array}$ \\
\hline \multirow{7}{*}{$\begin{array}{c}\text { The proposed } \\
\text { CDSS }\end{array}$} & Medium, 1.0 & 39.76 & 15.25 & 100.00 & 67.47 & 0.00 & 100.00 & 84.34 & 7.14 & 100.00 & 27.71 & 4.76 & 100.00 \\
\hline & Medium, 0.8 & 61.45 & 45.76 & 100.00 & 75.90 & 29.63 & 98.21 & 86.75 & 21.43 & 100.00 & 51.81 & 41.27 & 85.00 \\
\hline & Medium, 0.7 & 81.93 & 77.97 & 91.67 & 84.34 & 55.56 & 98.21 & 89.16 & 35.71 & 100.00 & 80.72 & 84.13 & 70.00 \\
\hline & Small, 1.0 & 87.95 & 86.44 & 91.67 & 87.95 & 66.67 & 98.21 & 89.16 & 35.71 & 100.00 & 86.75 & 92.06 & 70.00 \\
\hline & Small, 0.8 & 84.34 & 91.53 & 66.67 & 66.27 & 77.78 & 60.71 & 90.36 & 85.71 & 91.30 & 81.93 & 92.06 & 50.00 \\
\hline & Small, 0.7 & 78.31 & 93.22 & 41.67 & 56.63 & 77.78 & 46.43 & 89.16 & 85.71 & 89.86 & 81.93 & 93.65 & 45.00 \\
\hline & Small, 0.5 & 77.11 & 100.00 & 20.83 & 43.37 & 88.89 & 21.43 & 85.54 & 100.00 & 82.61 & 78.31 & 98.41 & 15.00 \\
\hline $\begin{array}{c}\text { Resident } \\
\text { geriatricians }\end{array}$ & & 66.27 & 61.02 & 79.17 & 92.77 & 77.78 & 100.00 & 86.75 & 42.86 & 95.65 & 89.16 & 95.24 & 70.00 \\
\hline
\end{tabular}

ACC—accuracy, TPR—true positive rate (sensitivity), TNR—true negative rate (specificity).

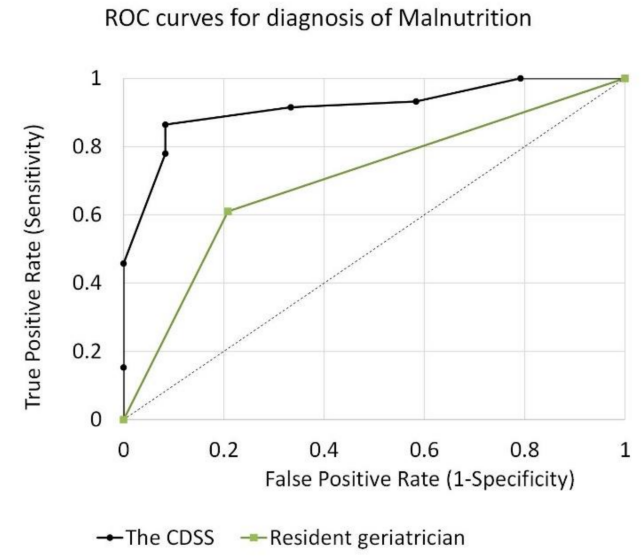

(a)

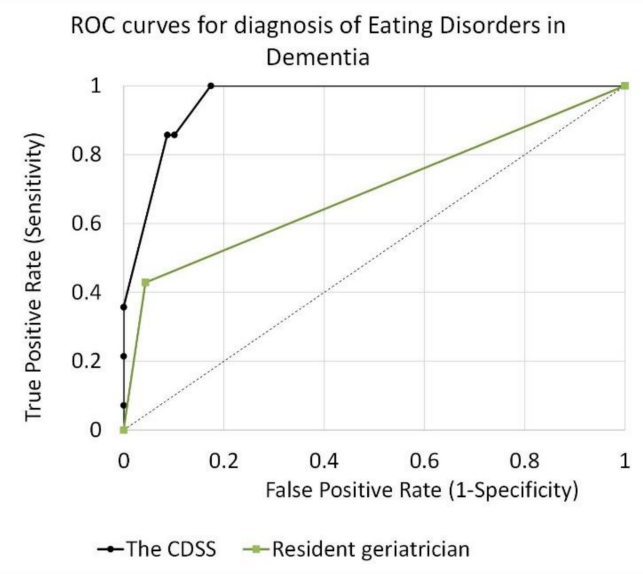

(c)

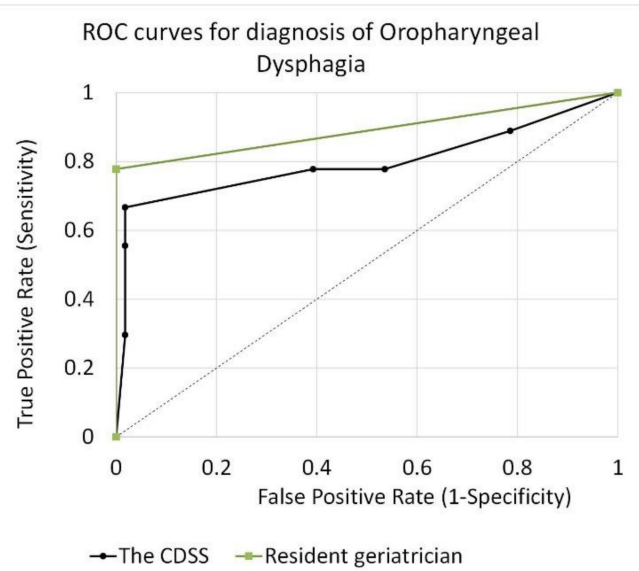

(b)

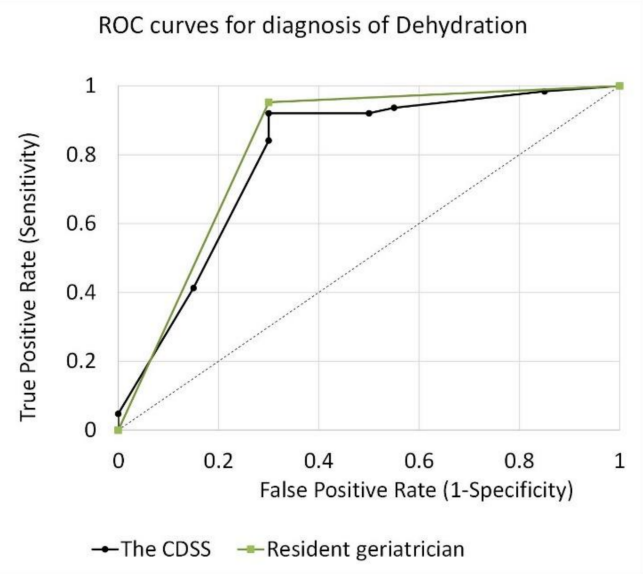

(d)

Figure 9. The ROC graphs of the proposed CDSS and resident geriatrician for diagnoses of nutrition-related syndromes: (a) the ROC curves for malnutrition; (b) the ROC curves for oropharyngeal dysphagia; (c) the ROC curves for eating disorders in dementia; (d) the ROC curves for dehydration. 
The highest prediction accuracy of $87.95 \%$ for diagnosing the malnutrition syndrome was achieved when a small risk level with 1.0 certainty was used as the threshold. As shown in Table 3 and Figure 9a, the accuracy of the proposed CDSS is much higher than the accuracy of the diagnoses of the resident geriatricians.

The highest prediction accuracy of $87.95 \%$ for diagnosing the oropharyngeal dysphagia syndrome was achieved when a small risk level with 1.0 certainty was used as the threshold. The accuracy of the proposed CDSS is only $4.82 \%$ lower than the accuracy of the diagnoses of the resident geriatricians. The highest prediction accuracy of $90.36 \%$ for the diagnosis of eating disorders in dementia was achieved when a small risk level with 0.8 certainty was used as the threshold. The accuracy of the proposed CDSS is $3.61 \%$ higher than the accuracy of the diagnoses of the resident geriatricians. Although the difference in accuracy is not large, the sensitivity of resident geriatricians is very low $(42.86 \%)$. This means that the CDSS has a much higher true positive prediction rate (ability to correctly identify the syndrome), while geriatricians are slightly better at detecting the absence of disorder. The highest prediction accuracy of $86.75 \%$ for the diagnosis of dehydration syndrome was achieved when a small risk level with 1.0 certainty was used as the threshold. The accuracy of the proposed CDSS is only $2.41 \%$ lower than the accuracy of the diagnoses of the resident geriatricians. The specificities are identical for both the CDSS and resident geriatricians.

The study found that the accuracy of the CDSS compared to the gold standard (the diagnosis made by expert geriatricians) is quite high, ranging from $86.75 \%$ to $90.36 \%$. Compared to the resident geriatricians, the CDSS is more accurate in diagnosing malnutrition and eating disorders in dementia (Figure 9a,c) and less accurate in diagnosing oropharyngeal dysphagia and dehydration (Figure 9b,d).

\section{Discussion}

This study appears to be the first to use explainable artificial intelligence (XAI) methods for the implementation of a clinical decision support system (CDSS) for the assessment of nutrition-related geriatric syndromes. As discussed in the Introduction Section, currently, geriatricians face difficulties in diagnosing the following nutrition-related disorders: malnutrition, oropharyngeal dysphagia, dehydration, and eating disorders in dementia. Usually, diagnosis is made using comprehensive geriatric assessment methods and laboratory examinations, which can include (but not necessarily) various questionnaires (e.g., oropharyngeal dysphagia). Even when used, questionnaires are based on inaccurate and subjective estimates of symptoms (usually a 1-4 point scale). Therefore, we have made the assumption that explainable artificial intelligence and fuzzy logic-based reasoning would be the most suitable methods to deal with this problem and to evaluate the relations between different syndromes, which cannot be seen directly from separate questionnaires. The proposed CDSS is based on a decision support model, which is presented in our previous work [6]. The model is based on the knowledge of expert geriatricians and is capable of assessing inaccurate input data and the degree of interrelation between symptoms and syndromes. The results of this study confirm that the previously proposed approach is suitable for practical application in CDSS. As a proof of concept, the CDSS prototype was created and tested with a large number of geriatric patients at the Lithuanian University of Health Sciences Kaunas Hospital. The comparison of the diagnoses provided by the CDSS and expert geriatricians shows that the accuracy of the CDSS is quite high: $87.95 \%$ in the case of malnutrition, $87.95 \%$ in the case of oropharyngeal dysphagia, $90.36 \%$ in the case of malnutrition eating disorders in dementia, $86.75 \%$ in the case of dehydration).

Although the accuracy of the CDSS diagnoses does not completely match that of the expert geriatrician, the results are quite promising because the CDSS is more accurate than resident geriatricians in diagnosing malnutrition and eating disorders in dementia, and only a few percent less accurate in diagnosing oropharyngeal dysphagia and dehydration. This confirms our next hypothesis that the proposed CDSS, while not a complete replacement for the expert physician, would be perfectly suited to help make a decision for less experienced 
physicians. The CDSS can be used as an additional tool useful not only for geriatricians but also for general practitioners. Since the qualification of general practitioners is in line with the experience of junior resident geriatricians, the developed CDSS will diagnose geriatric syndromes much more accurately and can be recommended as a support tool to help general practitioners (or other non-geriatricians) identify geriatric disorders.

One of the key benefits of XAI-based CDSS is that physicians can easily verify why and on what basis the CDSS provided a specific assessment of the syndrome and use it to determine the reasons for the discrepancy in the assessment. In this way, less experienced physicians can understand where their assessment errors are, and expert physicians can identify areas for improvement in the system. Despite the promising results of this study, there are several ways to increase the precision of the proposed CDSS. One direction for further research is applying a more sophisticated algorithm to convert the CDSS risk-certainty pairs into concrete diagnoses. Another direction can include improving questionnaires and including laboratory test results in the decision-making process. Research into solving this problem is already underway.

In general, there are many ways to improve the proposed nutrition-related geriatric syndromes assessment model and the CDSS. The proposed model and methodology for the assessment of syndromes have the advantage that it can be easily expanded with new symptoms and the possibility of identifying other syndromes and diseases or even extended with recommendations for further treatment.

\section{Conclusions}

This paper proposes a novel approach for the diagnosis of nutrition-related geriatric syndromes: malnutrition, oropharyngeal dysphagia, dehydration, and eating disorders in dementia. This approach is based on the original XAI-based health risks assessment model, which was implemented in the CDSS prototype tested under real conditions at the geriatric department of Lithuanian University of Health Sciences Kaunas Hospital. The diagnoses provided by the CDSS were compared with the diagnoses of resident physicians and expert physicians. The results of this study, involving 83 patients, show that the proposed CDSS can efficiently diagnose nutrition-related geriatric syndromes with high accuracy: $87.95 \%$ for malnutrition, $87.95 \%$ for oropharyngeal dysphagia, $90.36 \%$ for eating disorders in dementia, and $86.75 \%$ for dehydration. This confirms that the proposed XAI-based CDSS is an effective tool, allowing even less experienced medical personnel to make more accurate decisions faster and easier when the decision should be made using incomplete, uncertain, and subjective data. The experimental evaluation of the proposed CDSS proves that in some cases, the system makes even a more accurate decision than junior and senior resident geriatricians. Therefore, the CDSS could serve as a screening tool in clinical family medicine practice. If the risk of the syndrome is identified, the patient would be referred for an outpatient geriatric consultation or an inpatient geriatric examination (e.g., a day hospital).

Author Contributions: Conceptualization, V.P., R.J., and G.D.; data curation, G.D., Z.M., J.D., and V.K.; formal analysis, V.P., R.J., and G.D.; funding acquisition, A.L. and V.L.; investigation, G.D., J.K., and R.B.-P.; methodology, V.P., R.J., G.D., and J.K.; project administration, A.L. and V.L.; resources, V.L., Z.M., and V.K.; software, Z.M., J.D., and V.K.; supervision, A.L. and V.L.; validation, G.D.; visualization, A.L. and A.J.; writing-original draft preparation, V.P., R.J., A.L., and A.J.; writing—review and editing, V.P., R.J., G.D., A.L., A.J., and V.L. All authors have read and agreed to the published version of the manuscript.

Funding: This research was funded by the Research and Innovation Fund of Kaunas University of Technology (project grant no. PP34/2102) and the Research Fund of Lithuanian University of Health Sciences (project grant no. LSMU-08).

Institutional Review Board Statement: The study was conducted according to the guidelines of the Declaration of Helsinki and approved by the Regional Bioethics Committee at the Lithuanian University of Health Sciences (no. BE-2-106).

Informed Consent Statement: Informed consent was obtained from all subjects involved in the study. 
Data Availability Statement: The data presented in this study are available on request from the corresponding author. The data are not publicly available due to privacy and ethical reasons.

Conflicts of Interest: The authors declare no conflict of interest.

Appendix A

Short-Form Mini-Nutritional Assessment (MNA-SF) scale

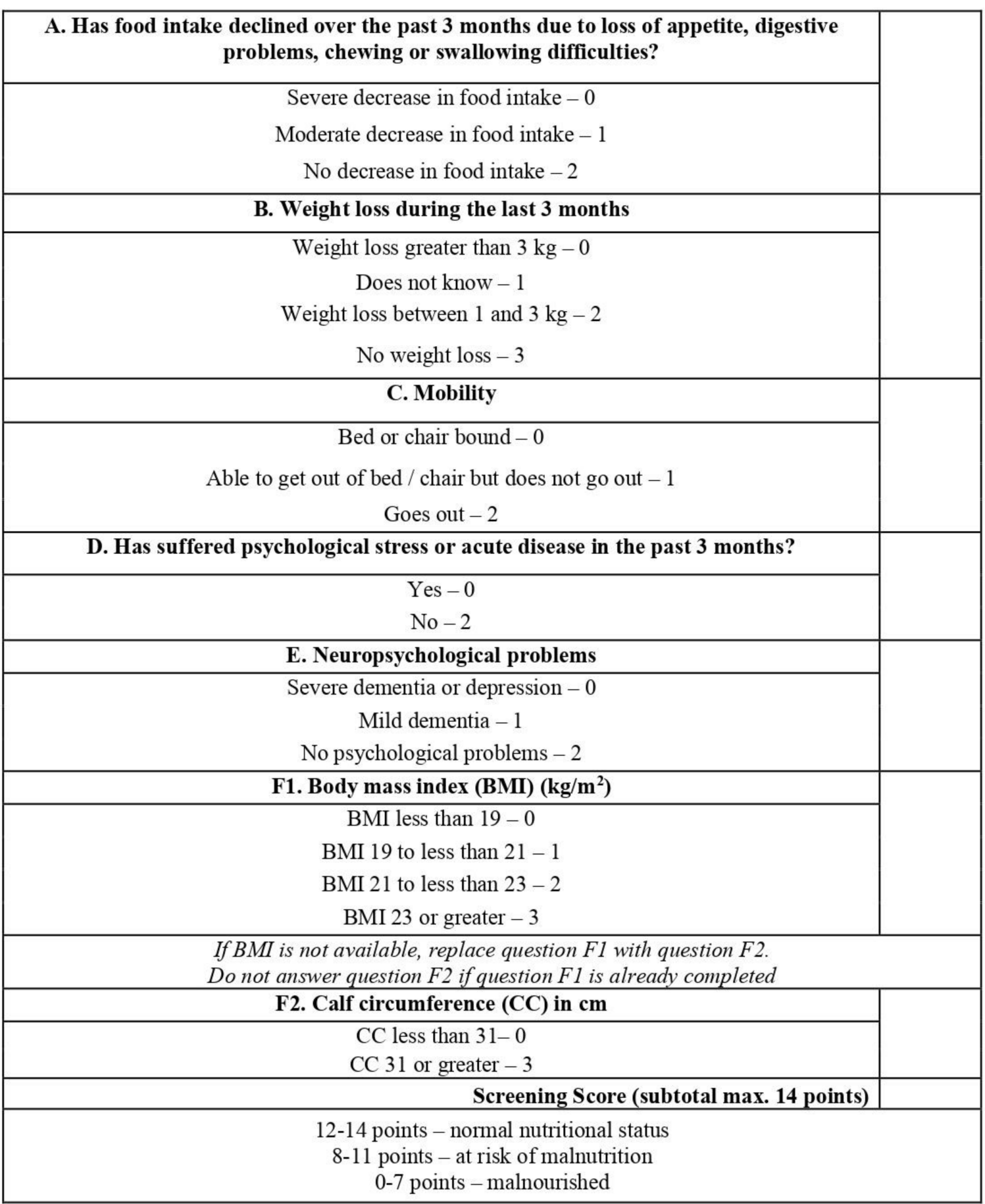

Figure A1. Short-Form Mini-Nutritional Assessment Scale. 


\section{EAT-10: A Swallowing Screening Tool}

\section{A. INSTRUCTIONS:}

Answer each question by writing the number of points in the boxes.

To what extent do you experience the following problems?

\begin{tabular}{|c|c|c|c|}
\hline $\begin{array}{c}\text { Swallowing problem has caused me to } \\
\text { lose weight }\end{array}$ & Score & Swallowing is painful & Score \\
\hline $0-$ no problem & & 0 - no problem & \\
\hline 1 & & 1 & \\
\hline 2 & & 2 & \\
\hline 3 & & 3 & \\
\hline 4 - severe problem & & 4 - severe problem & \\
\hline $\begin{array}{l}\text { My swallowing problem interferes with } \\
\text { my ability to go out for meals }\end{array}$ & Score & $\begin{array}{l}\text { The pleasure of eating is affected by } \\
\text { my swallowing }\end{array}$ & Score \\
\hline $0-$ no problem & & $0-$ no problem & \\
\hline 1 & & 1 & \\
\hline 2 & & 2 & \\
\hline 3 & & 3 & \\
\hline 4 - severe problem & & 4 - severe problem & \\
\hline Swallowing liquids takes extra effort & Score & $\begin{array}{c}\text { When I swallow food sticks in my } \\
\text { throat }\end{array}$ & Score \\
\hline 0 - no problem & & $0-$ no problem & \\
\hline 1 & & 1 & \\
\hline 2 & & 2 & \\
\hline 3 & & 3 & \\
\hline 4 - severe problem & & 4 - severe problem & \\
\hline Swallowing solids takes extra effort & Score & I cough when I eat & Score \\
\hline $0-$ no problem & & $0-$ no problem & \\
\hline 1 & & 1 & \\
\hline 2 & & 2 & \\
\hline 3 & & 3 & \\
\hline 4 - severe problem & & 4 - severe problem & \\
\hline Swallowing pills takes extra effort & Score & Swallowing is stressful & Score \\
\hline $0-$ no problem & & $0-$ no problem & \\
\hline 1 & & 1 & \\
\hline 2 & & 2 & \\
\hline 3 & & 3 & \\
\hline 4 - severe problem & & 4 - severe problem & \\
\hline
\end{tabular}

\section{B. Scoring}

Add up the number of points and write your total score in the boxes.

Total Score (max. 40 points)

Figure A2. Eating Assessment Tool EAT-10. 
Edinburgh Feeding Evaluation in Dementia Questionnaire (EdFED-Q)

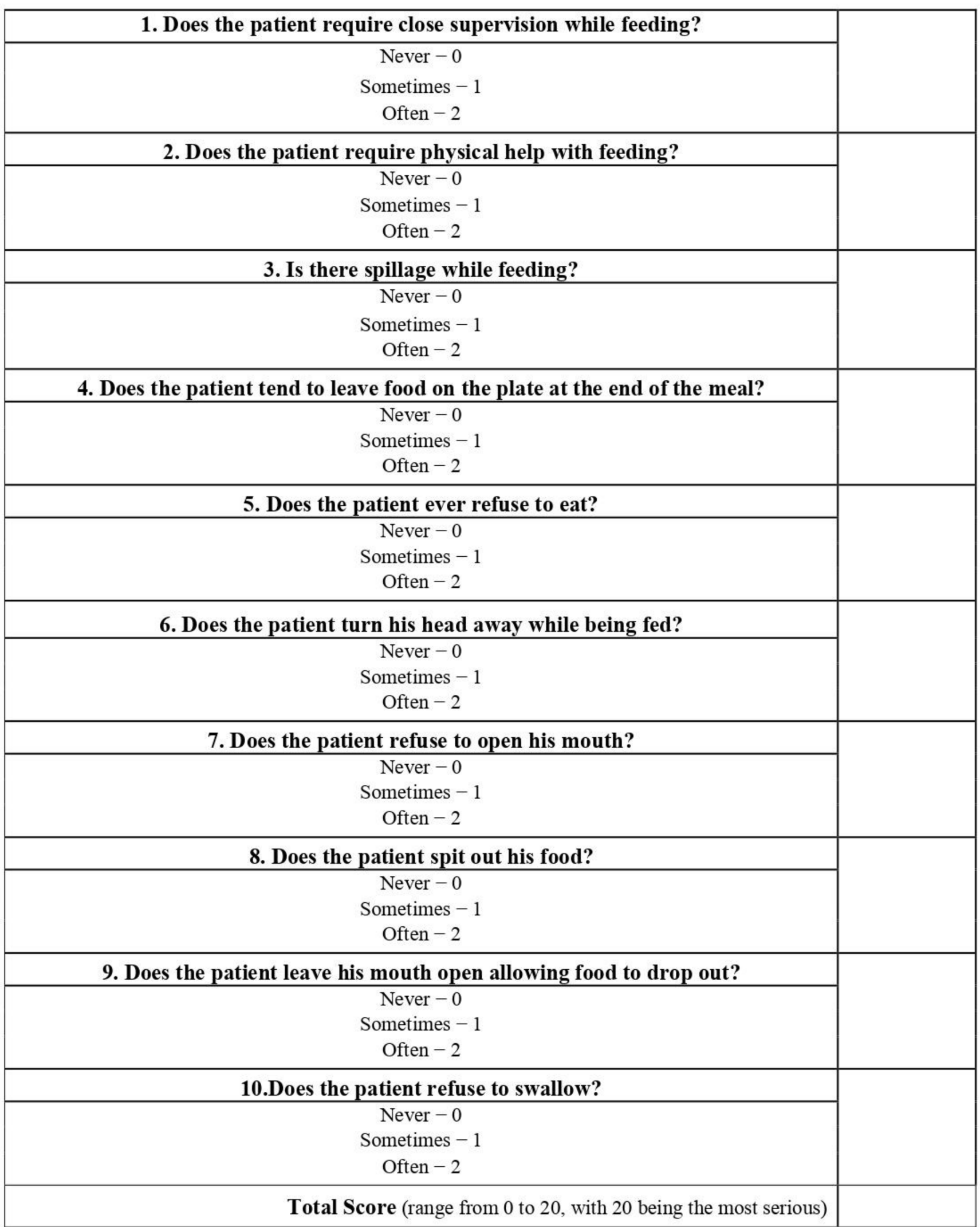

Figure A3. Edinburgh Feeding Evaluation in Dementia Scale. 


\section{Dehydration Assessment}

\begin{tabular}{|c|c|}
\hline 1. Do you have thirst? & \\
\hline $\begin{array}{l}0 \text { - no problem, 1- little problem, } 2 \text { - medium problem, } 3 \text { - big problem, } \\
4 \text { - severe problem }\end{array}$ & \\
\hline 2. Do you feel muscle weakness? & \\
\hline $\begin{array}{c}0 \text { - no problem, 1- little problem, } 2 \text { - medium problem, } 3 \text { - big problem, } \\
4 \text { - severe problem }\end{array}$ & \\
\hline 3. Do you have headache? & \\
\hline $\begin{array}{c}0 \text { - no problem, 1- little problem, } 2 \text { - medium problem, } 3 \text { - big problem, } \\
4 \text { - severe problem }\end{array}$ & \\
\hline 4. Do you feel anxiety? & \\
\hline $\begin{array}{l}0 \text { - no problem, 1- little problem, } 2 \text { - medium problem, } 3 \text { - big problem, } \\
4 \text { - severe problem }\end{array}$ & \\
\hline 5. Did your tongue and mouth mucosa become dry? & \\
\hline $\begin{array}{c}0 \text { - no problem, 1- little problem, } 2 \text { - medium problem, } 3 \text { - big problem, } \\
4 \text { - severe problem }\end{array}$ & \\
\hline $\begin{array}{l}\text { 6. Did you lose weight? } \\
\end{array}$ & \\
\hline $\begin{array}{l}0 \text { - no problem, 1- little problem, } 2 \text { - medium problem, } 3 \text { - big problem, } \\
4 \text { - severe problem }\end{array}$ & \\
\hline 7. Did the amount of urine decreased? & \\
\hline $\begin{array}{l}0 \text { - no problem, 1- little problem, } 2 \text { - medium problem, } 3 \text { - big problem, } \\
4 \text { - severe problem }\end{array}$ & \\
\hline 8. Has the amount of stool decreased and they became harder? & \\
\hline $\begin{array}{l}0 \text { - no problem, 1- little problem, } 2 \text { - medium problem, } 3 \text { - big problem, } \\
4 \text { - severe problem }\end{array}$ & \\
\hline 9. Did the heart rate increase? & \\
\hline $\begin{array}{c}0 \text { - no problem, 1- little problem, } 2 \text { - medium problem, } 3 \text { - big problem, } \\
4 \text { - severe problem }\end{array}$ & \\
\hline 10. Does standing up lower blood pressure and increase heart rate? & \\
\hline $\begin{array}{c}0 \text { - no problem, 1- little problem, } 2 \text { - medium problem, } 3 \text { - big problem, } \\
4 \text { - severe problem }\end{array}$ & \\
\hline Total Score (max. 40 points) & \\
\hline $\begin{array}{c}0-5-\text { normal } \\
6-10-\text { at risk of dehydration } \\
11-40-\text { dehydration }\end{array}$ & \\
\hline
\end{tabular}

Figure A4. Dehydration questionnaire.

\section{References}

1. Volkert, D.; Beck, A.M.; Cederholm, T.; Cruz-Jentoft, A.; Goisser, S.; Hooper, L.; Kiesswetter, E.; Maggio, M.; Raynaud-Simon, A.; Sieber, C.C.; et al. ESPEN Guideline on Clinical Nutrition and Hydration in Geriatrics. Clin. Nutr. 2019, 38, 10-47. [CrossRef] [PubMed]

2. Stockdell, R.; Amella, E.J. The Edinburgh Feeding Evaluation in Dementia Scale: Determining How Much Help People with Dementia Need at Mealtime. AJN Am. J. Nurs. 2008, 108, 46-54. [CrossRef]

3. Norman, K.; Haß, U.; Pirlich, M. Malnutrition in Older Adults-Recent Advances and Remaining Challenges. Nutrients 2021, 13, 2764. [CrossRef] [PubMed] 
4. Govindaraju, T.; Sahle, B.; McCaffrey, T.; McNeil, J.; Owen, A. Dietary Patterns and Quality of Life in Older Adults: A Systematic Review. Nutrients 2018, 10, 971. [CrossRef] [PubMed]

5. Browne, S.; Kelly, L.; Geraghty, A.A.; Reynolds, C.M.E.; McBean, L.; McCallum, K.; Clyne, B.; Bury, G.; Perrotta, C.; Kennelly, S.; et al. Healthcare Professionals' Perceptions of Malnutrition Management and Oral Nutritional Supplement Prescribing in the Community: A Qualitative Study. Clin. Nutr. ESPEN 2021, 44, 415-423. [CrossRef]

6. Petrauskas, V.; Damuleviciene, G.; Dobrovolskis, A.; Dovydaitis, J.; Janaviciute, A.; Jasinevicius, R.; Kazanavicius, E.; Knasiene, J.; Lesauskaite, V.; Liutkevicius, A. XAI-Based Medical Decision Support System Model. IJSRP 2020, 10, 598-607. [CrossRef]

7. Ali, L.; Khan, S.U.; Golilarz, N.A.; Yakubu, I.; Qasim, I.; Noor, A.; Nour, R. A Feature-Driven Decision Support System for Heart Failure Prediction Based on $\chi 2$ Statistical Model and Gaussian Naive Bayes. Comput. Math. Methods Med. 2019, 2019, 1-8. [CrossRef] [PubMed]

8. Casalino, G.; Castellano, G.; Castiello, C.; Pasquadibisceglie, V.; Zaza, G. A Fuzzy Rule-Based Decision Support System for Cardiovascular Risk Assessment. In Fuzzy Logic and Applications; Fullér, R., Giove, S., Masulli, F., Eds.; Lecture Notes in Computer Science; Springer International Publishing: Cham, Switzerland, 2019; Volume 11291, pp. 97-108. ISBN 978-3-030-12543-1.

9. Apostolopoulos, I.D.; Groumpos, P.P.; Apostolopoulos, D.I. A Medical Decision Support System for the Prediction of the Coronary Artery Disease Using Fuzzy Cognitive Maps. In Creativity in Intelligent Technologies and Data Science; Kravets, A., Shcherbakov, M., Kultsova, M., Groumpos, P., Eds.; Communications in Computer and Information Science; Springer International Publishing: Cham, Switzerland, 2017; Volume 754, pp. 269-283. ISBN 978-3-319-65550-5.

10. Kishore, A.H.N.; Jayanthi, V.E. Neuro-Fuzzy Based Medical Decision Support System for Coronary Artery Disease Diagnosis and Risk Level Prediction. J Comput. Theor. Nanosci. 2018, 15, 1027-1037. [CrossRef]

11. Hamedan, F.; Orooji, A.; Sanadgol, H.; Sheikhtaheri, A. Clinical Decision Support System to Predict Chronic Kidney Disease: A Fuzzy Expert System Approach. Int. J. Med. Inform. 2020, 138, 104134. [CrossRef] [PubMed]

12. Malmir, B.; Amini, M.; Chang, S.I. A Medical Decision Support System for Disease Diagnosis under Uncertainty. Expert Syst. Appl. 2017, 88, 95-108. [CrossRef]

13. El-Sappagh, S.; Alonso, J.M.; Ali, F.; Ali, A.; Jang, J.-H.; Kwak, K.-S. An Ontology-Based Interpretable Fuzzy Decision Support System for Diabetes Diagnosis. IEEE Access 2018, 6, 37371-37394. [CrossRef]

14. Spänig, S.; Emberger-Klein, A.; Sowa, J.-P.; Canbay, A.; Menrad, K.; Heider, D. The Virtual Doctor: An Interactive ClinicalDecision-Support System Based on Deep Learning for Non-Invasive Prediction of Diabetes. Artif. Intell. Med. 2019, $100,101706$. [CrossRef] [PubMed]

15. Lee, S.J.; Yu, S.H.; Kim, Y.; Kim, J.K.; Hong, J.H.; Kim, C.-S.; Seo, S.I.; Byun, S.-S.; Jeong, C.W.; Lee, J.Y.; et al. Prediction System for Prostate Cancer Recurrence Using Machine Learning. Appl. Sci. 2020, 10, 1333. [CrossRef]

16. Mazo, C.; Kearns, C.; Mooney, C.; Gallagher, W.M. Clinical Decision Support Systems in Breast Cancer: A Systematic Review. Cancers 2020, 12, 369. [CrossRef] [PubMed]

17. Bucholc, M.; Ding, X.; Wang, H.; Glass, D.H.; Wang, H.; Prasad, G.; Maguire, L.P.; Bjourson, A.J.; McClean, P.L.; Todd, S.; et al. A Practical Computerized Decision Support System for Predicting the Severity of Alzheimer's Disease of an Individual. Expert Syst. Appl. 2019, 130, 157-171. [CrossRef]

18. Papageorgiou, E.I.; Billis, A.S.; Frantzidis, C.; Konstantinidis, E.I.; Bamidis, P.D. A Preliminary Fuzzy Cognitive Map-Based Desicion Support Tool for Geriatric Depression Assessment. In Proceedings of the 2013 IEEE International Conference on Fuzzy Systems (FUZZ-IEEE), Hyderabad, India, 7-10 July 2013; pp. 1-8.

19. Tolonen, A.; Rhodius-Meester, H.F.M.; Bruun, M.; Koikkalainen, J.; Barkhof, F.; Lemstra, A.W.; Koene, T.; Scheltens, P.; Teunissen, C.E.; Tong, T.; et al. Data-Driven Differential Diagnosis of Dementia Using Multiclass Disease State Index Classifier. Front. Aging Neurosci. 2018, 10, 111. [CrossRef]

20. Suárez-Araujo, C.P.; García Báez, P.; Cabrera-León, Y.; Prochazka, A.; Rodríguez Espinosa, N.; Fernández Viadero, C.; for the Alzheimer's Disease Neuroimaging Initiative. A Real-Time Clinical Decision Support System, for Mild Cognitive Impairment Detection, Based on a Hybrid Neural Architecture. Comput. Math. Methods Med. 2021, 2021, 1-9. [CrossRef]

21. Bruun, M.; Frederiksen, K.S.; Rhodius-Meester, H.F.M.; Baroni, M.; Gjerum, L.; Koikkalainen, J.; Urhemaa, T.; Tolonen, A.; van Gils, M.; Rueckert, D.; et al. Impact of a Clinical Decision Support Tool on Prediction of Progression in Early-Stage Dementia: A Prospective Validation Study. Alz Res. Ther. 2019, 11, 25. [CrossRef]

22. Amirkhani, A.; Papageorgiou, E.I.; Mosavi, M.R.; Mohammadi, K. A Novel Medical Decision Support System Based on Fuzzy Cognitive Maps Enhanced by Intuitive and Learning Capabilities for Modeling Uncertainty. Appl. Math. Comput. 2018, 337, 562-582. [CrossRef]

23. Mencar, C.; Alonso, J.M. Paving the Way to Explainable Artificial Intelligence with Fuzzy Modeling: Tutorial. In Fuzzy Logic and Applications; Fullér, R., Giove, S., Masulli, F., Eds.; Lecture Notes in Computer Science; Springer International Publishing: Cham, Switzerland, 2019; Volume 11291, pp. 215-227. ISBN 978-3-030-12543-1.

24. Rubenstein, L.Z.; Harker, J.O.; Salva, A.; Guigoz, Y.; Vellas, B. Screening for Undernutrition in Geriatric Practice: Developing the Short-Form Mini-Nutritional Assessment (MNA-SF). J. Gerontol. Ser. A Biol. Sci. Med Sci. 2001, 56, M366-M372. [CrossRef]

25. Belafsky, P.C.; Mouadeb, D.A.; Rees, C.J.; Pryor, J.C.; Postma, G.N.; Allen, J.; Leonard, R.J. Validity and Reliability of the Eating Assessment Tool (EAT-10). Ann. Otol. Rhinol. Laryngol. 2008, 117, 919-924. [CrossRef] [PubMed]

26. Watson, R.; MacDonald, J.; McReady, T. The Edinburgh Feeding Evaluation in Dementia Scale \#2 (EdFED \#2): Inter- and Intra-Rater Reliability. Clin. Eff. Nurs. 2001, 5, 184-186. [CrossRef] 
27. Jasinevicius, R.; Petrauskas, V. Fuzzy Expert Maps: The New Approach. In Proceedings of the 2008 IEEE International Conference on Fuzzy Systems (IEEE World Congress on Computational Intelligence), Hong Kong, China, 1-6 June 2008; pp. $1511-1517$.

28. Petrauskas, V.; Jasinevicius, R.; Kazanavicius, E.; Meskauskas, Z. Concept of a System Using a Dynamic SWOT Analysis Network for Fuzzy Control of Risk in Complex Environments. Math. Comput. Sci. 2020, 5, 42. [CrossRef]

29. Baijens, L.W.; Clavé, P.; Cras, P.; Ekberg, O.; Forster, A.; Kolb, G.; Leners, J.C.; Masiero, S.; Mateos del Nozal, J.; Ortega, O.; et al. European Society for Swallowing Disorders-European Union Geriatric Medicine Society White Paper: Oropharyngeal Dysphagia as a Geriatric Syndrome. Clin. Interv. Aging 2016, 11, 1403-1428. [CrossRef] [PubMed] 